\title{
Recurrent perforation of the ileum
}

\author{
J. D. CORMACK, M. A. MELSOM, AND J. G. WINWICK \\ From the Pathology Laboratory, British Military Hospital, Rinteln, West Germany
}

SUMMARY Ulceration of the small intestine is rare, perforation of such ulcers is also rare, and repeated perforation is still rarer. A case with this history is presented, and the aetiology of such cases briefly discussed, as the current literature gives a varied emphasis regarding the important causes of ulceration of the small intestine.

\section{Case History}

A 34-year-old soldier presented on 28 August 1968 with a four-day history of increasingly severe abdominal pain. On examination pain, guarding, and rebound tenderness in the right iliac fossa were noted. The previous history revealed recurrent indigestion for years with a small haematemesis in 1960. A barium meal had shown duodenal cap deformity. Six months before admission the patient had an acute illness, with vomiting, which settled over one week. He had then had diarrhoea, lower abdominal pain, and tenesmus intermittently. His appetite was poor and his weight fell by 2 stones over the six months. Diagnoses considered preoperatively were perforation of a duodenal ulcer or a ruptured appendix.

At laparotomy a perforation was found in one of two enlarged Peyer's patches close to the ileocaecal junction. The bowel was otherwise normal with no evidence of obstruction. Enlarged, soft mesenteric glands were noted. Simple closure by oversewing the perforation was performed.

Postoperative progress was difficult. Ileus persisted for five days, and there was a low-grade fever. On the tenth day subacute obstruction occurred, which settled with conservative management. During this period, a further weight loss took place. The patient's progress then improved, his appetite recovered, the bowel rhythm became normal, and a weight gain of 15 pounds was noted.

Received for publication 2 December 1969.
Investigations included simple haematology, latex tests for RA and LE factors, electrolytes, blood urea, serum amylase, serum proteins and electrophoresis, and serum transaminases, all being normal. The morphology of the bone marrow was also normal, as were the results of all investigations of bowel function. These included stool culture, xylose absorption, and faecal fat excretion ( $4.8 \mathrm{~g} /$ day). The serum $B_{12}$ was $250 \mu \mu \mathrm{g}$. Occult blood tests were predominantly negative, only three positive results occurring closely after the operation. Bacteriological investigation of swabs from the perforation and peritoneal exudate showed no significant organisms and repeated blood cultures were sterile. The Widal tests on repeated sera showed no significant rise in titre, and barium meal and enema examinations added no helpful information.

On 19 December 1968 the patient was readmitted having been well until two months previously, when he developed intermittent colicky abdominal pains, loss of appetite, and altered bowel rhythm, mainly constipation, but with bouts of loose, watery stools. Before admission the pains had increased in severity and localized to the right iliac fossa. Examination revealed a distended lower abdomen with marked right iliac fossa tenderness, guarding, and rebound tenderness. At laparotomy a perforated ulcer was found $16 \mathrm{~cm}$ from the ileocaeal valve. The proximal bowel was dilated and multiple adhesions were present. The ascending right colic artery was seen to be thrombosed extensively, and for this reason, the resection procedure carried 
out included a right hemicolectomy with end-toend ileocolic anastomosis. Postoperative progress was rapid and uneventful. In February 1969 the patient was without pain, eating normally, and feeling well. Further investigations carried out with normal results included fluorescent antibody titres to $E$. histolytica and screening the urine for excess 5HIAA.

\section{Pathology}

Macroscopic examination showed a dilated ileum proximal to the site of the perforation. The ulcer was at $90^{\circ}$ to the mesenteric border, around which were three mucosal polyps. The adjacent serosal surface was inflamed.

Microscopic appearances showed a disruption of normal structure with fibrosis and inflammatory tissue, with pseudopolyp formation. In addition, there was sclerosis and thrombosis of small arteries in the submucosa and muscularis in relation to the perforation. Sections proximal to the site of perforation showed an unusual quantity of agyrophilic pigment in the mucosal inflammatory tissue. There was no histological evidence of neoplasia, tuberculosis, Crohn's disease, or amoebiasis.

\section{Discussion}

The causes of small bowel perforation are numerous and have recently been reviewed by Wayte and Helwig (1968). The clinical history of the present case eliminated trauma in all its forms, and the action of ingested drugs, such as potassium chloride, thiazide, steroids, and other drugs, as a cause of the perforation. The operative findings and subsequent histology eliminated foreign bodies, ectopic gastric mucosa, and neoplasia of either epithelial or mesodermal tissues. Histology excluded Crohn's disease, tuberculosis, sarcoid, amoebiasis, and typhoid. Bacteriological, serological, and clinical investigations relevant to these diseases were negative.

When the classical causes of perforation have been excluded, the remainder have been grouped as 'non-specific'. Recent authors have discussed the aetiology of these ulcers. Goehrs, Morlock, and Dockerty (1957); Teicher, Arlen, Muehlbauer, and Allen (1963); Golding and Goodwill (1965); Gillespie (1966); Watt, Watson, and Haase (1967); and Wayte and Helwig (1968) have all referred to vascular mechanisms in this role, with ulceration a common result.

Goehrs et al (1957) reviewed the series of nonspecific small-intestinal ulcers from the Mayo Clinic, and commented on the presence of vascular thrombosis in the submucosa. Teicher et al (1963) reported four cases of small-intestinal ulceration, in one of which the ulcer had perforated. They believed that the available histology suggested the ulceration to be secondary to a vascular change in the mesentery and felt that the varying age distribution implied varying pathological processes in the vessels. Gillespie (1966) described a case of multiple ulcers in the jejunum and ileum, with perforation, which was attributable to multiple emboli. Golding and Goodwill (1965) reported ileal perforation in a case of rheumatoid arthritis, where the patient had not received steroids, and they also demonstrated a periarteritis in the vessels in the submucosa. They reviewed similar cases of such arteritis occurring in collagenous disease. Watt et al (1967) reported five cases of extensive vascular disease, and, using lateral aortography, showed abnormalities of the coeliac axis or the mesenteric arteries. The features of these cases included malabsorption in four patients and marked weight loss and intestinal angina. In these cases of disease of the larger arteries local ulceration was not reported.

Cases with a vascular aetiology appear to fall into two groups. The first is associated with widespread atherosclerosis, as in the patients described by Watt et al (1967), two of whom died of coronary thrombosis. This group is characterized by postprandial pain, ie, intestinal angina, weight loss, malabsorption, and not by local ulceration. The second group had ulceration associated with thrombosis of small vessels, frequently with no other evidence of vascular disease, although collagen disease with its arteritis has produced a similar picture. Such cases usually presented with recurrent colic and partial obstruction.

The present patient, who had repeated episodes of perforation, also had symptoms consistent with subacute obstruction, although bleeding was not a factor, as it was in the case reported by Shah (1968).

The progress and prognosis for cases with ulceration and thrombosis appear good following resection of the abnormal segment. In the present case the good postoperative course after the second perforation contrasted with that after the first, when an infective cause such as typhoid was thought likely. Goehrs et al (1957) reported the follow-up of 20 cases of resection from 18 months to 25 years. Thirteen were asymptomatic and a further seven had only minor discomfort.

Other cases of small bowel ulceration are not clearly related to vascular abnormalities (Shah, 1968). However, in their review, Wayte and Helwig (1968) believe that the terminal ileum has a precarious blood supply and that vascular factors not easily demonstrable may be responsible.

The management of cases of small-bowel ulceration will be largely influenced by the manner of presentation. Instances of subacute obstruction and bleeding would be accepted as indi- 
cations for resection, while presentation with perforation, when the aetiology is in doubt and the risk of peritoneal contamination is high, may best be managed by simple closure. The present report emphasizes the risk of recurrence in cases with a vascular aetiology, and in such cases earlier, planned resection should be considered.

Acknowledgments aremade to Lieutenant-Colonel W. C. Moffat, FRCS, RAMC, under whose care the patient was first admitted, and to Colonel S. E. Large, MD, FRCP, L/RAMC, for his helpful criticism.

\section{References}

Gillepsie; G. (1966). Unusual case of multiple spontaneous perforation of small bowel. Brit. med. J., 2, 155-156.

Goehrs, H. R., Morlock, C. G., and Dockerty, M. B. (1957) Primary non-specific ulcers of the small intestine. Proc. Mayo Clin., 32, 351-353.

Golding, D. N., and Goodwill, C. J. (1965). Ileal perforation and acute peripheral neuropathy in rheumatoid arthritis Postgrad. med. J., 41, 27-30.

Shah, M. J. (1968). Primary non-specific ulcer of ileum presentin with massive rectal haemorrhage. Brit. med. J., 3, 474.

Teicher, I., Arlen, M., Muehlbauer, M., and Allen, A. C. (1963). The clinical-pathological spectrum of primary ulcers of the small intestine. Surg. Gynec. Obstet., 116, 196-202.

Watt, J. K., Watson, W. C., and Haase, S. (1967). Chronic intestinal ischaemia. Brit. med. J., 3, 199-202.

Wayte, D. M., and Helwig, E. B. (1968). Small-bowel ulceration -iatrogenic or multifactorial origin? Amer. J. clin. Path. 49, 26-40. 